\title{
Chromosomal Preimplantation Genetic Diagnosis: 25 Years and Counting
}

\author{
Kathryn D. Sanders ${ }^{1,2} \cdot$ Darren K. Griffin ${ }^{1}$
}

Received: 7 March 2017/Accepted: 21 March 2017/Published online: 22 April 2017

(c) The Author(s) 2017. This article is an open access publication

\begin{abstract}
Preimplantation genetic diagnosis (PGD), first successfully carried out in humans in the early 1990s, initially involved the PCR sexing of embryos by Y- (and later also X-) chromosome specific detection. Because of the problems relating to misdiagnosis and contamination of this technology however the PCR based test was superseded by a FISH-based approach involving X and Y specific probes. Sexing by FISH heralded translocation screening, which was shortly followed by preimplantation genetic screening (PGS) for Aneuploidy. Aneuploidy is widely accepted to be the leading cause of implantation failure in assisted reproductive technology (ART) and a major contributor to miscarriage, especially in women of advanced maternal age. PGS (AKA PGD for aneuploidy PGD-A) has had a chequered history, with conflicting lines of evidence for and against its use. The current practice of trophectoderm biopsy followed by array CGH or next generation sequencing is gaining in popularity however as evidence for its efficacy grows. PGS has the potential to identify viable embryos that can be transferred thereby reducing the chances of traumatic failed IVF cycles, miscarriage or congenital abnormalities and facilitating the quickest time to live birth of chromosomally normal offspring. In parallel to chromosomal diagnoses, technology for PGD has allowed for improvements in accuracy and efficiency of the genetic screening of embryos for monogenic disorders. The number of genetic conditions available for screening has increased since the early days of PGD, with the human
\end{abstract}

Darren K. Griffin

d.k.griffin@kent.ac.uk

1 School of Biosciences, University of Kent, Canterbury CT2 7NJ, UK

2 Genesis Genetics Ltd, London Biosciences Innovation Centre, Royal College Street, London NW1 0NH, UK fertilization and embryology authority currently licensing 419 conditions in the UK [1]. A novel technique known as karyomapping that involves SNP chip screening and tracing inherited chromosomal haploblocks is now licensed for the PGD detection of monogenic disorders. Its potential for the universal detection of chromosomal and monogenic disorders simultaneously however, has yet to be realized.

Keywords Preimplantation genetic screening . Preimplantation genetic diagnosis - Aneuploidy · Fluorescent in situ hybridization - Karyomapping - Next generation sequencing

\section{Introduction}

Preimplantation Genetic Diagnosis PGD is essentially a medical intervention designed to minimize the chances of transfer of genetically abnormal embryos in an IVF setting. Its primary utility is to help families at risk of transmitting genetic disorders conceive a normal child and/or to improve IVF success rates by the selective implantation of chromosomally normal embryos. Typically, the process involves referral and genetic counselling for the nature of the specific problem, standard IVF, embryo biopsy, genetic diagnosis of the biopsied cells then selective transfer of embryo(s) thought to be genetically normal.

\section{The First PGD Cases}

The first recorded PGD case in model species was a chromosomal one, performed to control sex ratio in rabbits [2]. Gardner and Edwards successfully assessed trophoblast fragments for inactive sex chromatin (Barr body) 
in females and thereby accurately determined the sex of blastocysts. Application of this technology to humans clearly had the potential to screen for X-linked recessive diseases before implantation of an embryo, avoiding invasive prenatal assessments and the possibility of a difficult decision deciding whether to terminate. Following the development of in vitro fertilization (IVF) in 1978 [3], clinical progress in this area became possible and thus, in 1990, the first human embryos underwent blastomere biopsy and the sex was established by PCR amplifying a Y-specific repeat sequence. The unaffected female embryos having two copies of the $\mathrm{X}$ chromosome and thus no amplified signal lacking the $\mathrm{Y}$ were transferred, resulting in successful pregnancy and healthy live birth free from the X-linked condition [4]. This led the way to PGD in other monogenic conditions such as cystic fibrosis, which was successfully achieved in 1992 [5]. Early PGD used polymerase chain reaction (PCR) to amplify short fragments of the known affected region of DNA using nested primers; providing confirmation if the cell and thus embryo possessed the sequence which coded for the condition in question. Thereafter, for most of the history of PGD diagnoses were either monogenic, usually involving increasingly sophisticated variants of PCR, or chromosomal, initially involving fluorescent in situ hybridization (FISH), but later involving whole karyotype screening approaches. The purpose of this review is to concentrate on the chromosomal side of the diagnoses.

FISH was first introduced clinically in 1992 to sex embryos using probes specific to $\mathrm{X}$ and $\mathrm{Y}$ chromosomes $[6,7]$. It is thus 25 years since we performed the first chromosomal PGD cases. Later in 1993, the first aneuploidy screening cases using FISH were carried out, assessing chromosome copy numbers of the most common trisomies associated with live birth defects X, Y, 13, 18 and 21 [8, 9]. The number of chromosomes that could be screened simultaneously was limited by the colours of the probes: red, yellow, green, aqua and blue. PGS most commonly was used for patients undergoing IVF with the following indications: advanced maternal age (AMA), recurrent miscarriage, recurrent implantation failure and those with severe male factor infertility. By screening embryos to identify and transfer chromosomally normal embryos, IVF success rates and pregnancy outcomes should be improved.

\section{The Trouble with PGS}

Initial, retrospective, studies of PGS indicated that there was an increase in implantation rates and decrease in pregnancy loss following PGS with FISH [9, 10]. Several, randomized controlled trials (RCTs) challenged this however, showing either no significant improvement or a detrimental effect on successful outcomes of IVF [11, 12]. There are differing opinions regarding why these studies had varying outcomes. Firstly, there is concern that the process of embryo biopsy at the cleavage stage could have an adverse effect on the embryo, at this stage in embryo development there are normally 8 cells, removing one of these could reduce the success of the future development of that embryo. Related to this, the other remaining cells (and hence the developmental potential of the embryo) could be damaged in the biopsy process and this could be operatordependent. Therefore, the studies that saw a decrease in implantation rate when compared to standard IVF without PGS, have been criticized for inadvertently causing damage during the biopsy process in those embryos which were later transferred and failed to implant. Secondly, it is known that embryos can present some degree of chromosomal variation between cells or mosaicism. For some cases of PGS it is possible that the cell that is screened will present as chromosomally normal, where in fact the other cells are abnormal, creating a false negative result. Thirdly, another possible contributor is that with most PGS studies using FISH, not all chromosomes are analysed. The chromosomes that are screened may have appeared euploid, but those chromosomes that have not been screened could be aneuploid, resulting in the transfer of an abnormal embryo. The practice of PGS in the clinical setting ultimately declined following the publication of these RCTs. FISH in most clinical IVF cases is now not the method of choice, in part due to lack of confidence in the technique but also from the emergence of advanced technology which was subsequently applied to PGS.

\section{Trophectoderm Biopsy and Improved Methods for PGS and PGD}

Improved culture conditions leading to a greater number of embryos reaching the blastocyst stage in regular IVF presented an opportunity for an improved approach to PGS protocols. Trophectoderm biopsy on day 5 of embryo development was now an attractive option over the conventional blastomere biopsy on day 3. The advantage of trophectoderm biopsy is clear, by day 5 of embryo development there are many cells that make up both the inner cell mass (ICM) and the trophectoderm. Removing a few cells from the trophectoderm while leaving the ICM undisturbed in theory has the potential for less adverse effects on the developing embryo and the advantage of providing more cells for analysis, than blastomere biopsy. A study by Kokkali et al. [13] demonstrated an improved implantation rate with blastocyst biopsy over cleavage biopsy and subsequent studies support these findings [14]. 
More recently, it has been shown that trophectoderm biopsy can be more consistent and reproducible across different practitioners and clinics compared to blastomere biopsy [15].

Perhaps the major technical advance in our ability to screen biopsied cells for chromosome abnormalities was the development of whole genome amplification (WGA) $[16,17]$. This approach increases the amount of available DNA where only small amounts are initially available from single cells. WGA enables multiple tests to be carried out, for example, PGS and PGD simultaneously, while benefiting from an increase in accuracy and sensitivity. Another benefit is that WGA products can be stored for later subsequent analysis in the instance of test failure or to confirm findings. WGA enabled array comparative genomic hybridization (aCGH) for the analysis of all chromosome copy number. aCGH essentially compares the amplified DNA labelled in one fluorescent colour with known, normal DNA labelled in another colour simultaneously hybridized to a genome-wide microarray. Chromosome-by-chromosome ratio analysis gives an indication of cytogenetic gain or loss e.g., trisomy or monosomy. Randomized clinical trials suggest benefits for screening by aCGH in terms of the usual outcomes used to measure IVF success [18].

Another application of WGA was that multiplex PCR was successfully adapted for PGD, this allowed for the simultaneous analysis of linked markers to screen for monogenic conditions as well as aneuploidy for selected chromosomes. This permitted screening of multiple conditions, with greater accuracy as allele dropout (ADOwhere a heterozygous individual was erroneously called as homozygous due to allele specific amplification) presented less of an issue with this technique than that seen with earlier applications of PGD due to the fact that multiple loci could be screened. Human leukocyte antigen (HLA) typing, also known as saviour siblings, could also be combined with aneuploidy and monogenic PGD and was first successfully performed using PGD in 2000 [19]. This process establishes a pregnancy and live birth that is a HLA-match to an existing sibling, by selecting an embryo which is a HLA-match for transfer who can then be a stem cell transplant donor for their older brother or sister. As most couples requiring this form of PGD are of AMA there is therefore a potential benefit to be able to combine this with PGS [20].

\section{Is There Still a Problem with PGS?}

Despite improvements in technology there is still an ongoing debate regarding the effectiveness of PGS for improved implantation and ongoing pregnancy rates. There have been several studies that have shown an improvement when PGS is used. A systematic review by Lee et al. [21] where they combined the findings of 19 articles, which were comprised of 3 RCTs and 16 observational studies, showed that PGS overall had improved success rates when compared to morphology based embryo selection. While, RCTs are considered the best design for research, the nature of ART in the clinical setting makes it difficult to create studies that meet all the criteria of a RCT, for example patients will always want to be in the group with the best outcome, they may wish to switch groups during the study to get what they perceive to be the best outcome, this can skew results, but would be unethical not to let patients have a choice. There are also many more unknowns associated with this area of medicine, such as the complex interaction of the physiologies of two people (in order to produce a third). The comparison of different retrospective studies carried out in different clinics with varying approaches to ART and differing levels of biopsy practitioner skill can still play a big part in the variation of results presented. Ideally, all clinics would be uniform in their techniques to draw conclusive comparisons however this is not always practicable. It should also be kept in mind, in those cases where PGS does improve outcome, whether the cost implications associated with PGS match the increase in success rates. PGS techniques remain relatively high cost when added to a conventional IVF cycle. The effect on the patient however, is difficult to quantify. Couples undergoing IVF cycles with PGS may potentially avoid the transfer of an embryo which has a high chance of miscarriage, meaning that they will be able to progress to the next possibly successful cycle much quicker than if an aneuploid embryo is transferred, implanted and miscarried.

\section{Karyomapping and Next Generation Sequencing (NGS)}

Karyomapping, first developed in 2010 [22], is a method that uses the principles of linkage analysis and the inheritance of chromosomal haploblocks, in which the mother, father and a reference affected family member or grandparents are compared to map the origin of each chromosome inherited (and any crossovers between grandparental chromosomes). Karyomaps allow the tracking of affected genes that reside on these haploblocks, which can then subsequently be used for PGD to identify unaffected embryos before transfer. When applied to screening embryos this can also give valuable additional information to detect monosomy, uniparental disomy and meiotic trisomies. The karyomaps that are produced offer easy visualization of the chromosomes and present clearly where there is crossover of genetic material. Karyomapping has the advantage of allowing for diagnosis of genetic 
conditions, while simultaneously screening for chromosomal imbalances however its potential for the use in aneuploidy screening has yet to be realized [23]. This method has been made possible through whole genome sequencing, it is more commonly used with single nucleotide polymorphisms (SNPs) chip technology but can also be used alongside next generation sequencing (NGS). To date however, NGS has primarily been used for aneuploidy screening.

NGS is a high resolution whole genome sequencing technology that allows for the processing of samples at high throughput with a high level of accuracy. Recent studies show NGS to have $100 \%$ specificity and sensitivity making it superior to aCGH for PGS [24]. NGS provides the ability to run samples simultaneously which gives the potential to make this technology lower cost and quicker than that seen with aCGH. It has also has the potential to identify small copy number variations (CNVs), which can affect embryo development and result in severe birth defects [25].

\section{The Impact of Cryopreservation and "Freeze All" Strategies on PGS}

ART has seen improvement in embryo cryopreservation techniques. In PGS, there has been a shift towards the cryopreservation of embryos and transfer later, when the status of embryos has been confirmed. Increased pregnancy success rates when screening for aneuploidy, have been attributed to the advancement of PGS technologies, such as aCGH and NGS. However, studies have shown there to be an improvement associated with frozen embryo transfer (FET) compared to fresh embryo transfer. This improvement is thought to be due to ovarian stimulation having a detrimental effect on the endometrium, which lowers implantation rate. This stimulation is not encountered during a frozen embryo transfer and could therefore lead to higher implantation rates [26]. Further research is required to determine if PGS is offering increased pregnancy success rates in addition to those seen with FET.

\section{What We Have Learnt from Research into PGS?}

A greater understanding of meiosis, crossing over and molecular biology has led to improvements in PGD and PGS. Similarly however by studying the chromosomal aspects of PGS we can understand the basic biology of early human development better. One example is the incidence of mosaicism, previously it was believed that mosaicism was very rare and that in the case of trisomies it would be throughout all the cells in an embryo. Indeed some studies have suggested that most human embryos are aneuploid and mosaic. In a recent study by Maxwell et al. [27], WGA products that had initially been assessed using aCGH were retested using NGS. Embryos that had originally been determined to be euploid by aCGH were found to be mosaic by NGS, some of these mosaic embryos that had been transferred resulted in miscarriage, this provided an explanation as to why these pregnancies subsequently failed. However, other embryos that had been found to be mosaic through retesting using NGS were found to have resulted in a healthy live birth. Two per cent of all normal pregnancies are post zygotically mosaic; therefore, caution is required when considering mosaic embryos at the time of transfer. Further research is required to ascertain the prevalence of mosaic cells in embryos and the implications on pregnancy outcome.

We have learnt that aneuploidy in embryos is commonplace but we are learning more about the effect of abnormalities on embryo development. It has for instance been found that aneuploidy rates are lower at day 5 than day 3 [14], raising the possibility that some aneuploidies are corrected or selected against between day 3 and day 5. It has also been suggested that meiotic abnormality, for example in patients who are Robertsonian translocation carriers; can affect the segregation of other structurally normal chromosomes, this may results in an interchromosomal effect on the subsequent mitotic divisions and thus a higher abnormality rate in these patients than in other unaffected patients [28]. It is beyond the scope of this review to cover all the biological implications of PGS findings, however the fact remains that it is a unique, fundamental system to study and much further research is still needed to address basic biological questions. For instance, we are still not entirely sure of the precise incidence, cell by cell, of aneuploidy in blastocyst embryos and, indeed, if a small amount of abnormality is commonplace in most embryos.

\section{The Future and Conclusions}

PGD and PGS have both come a long way since their first use in the early 1990s. We now believe that most embryos are aneuploid and that by transferring embryos that are aneuploid it is likely to result in failure to implant, miscarriage or the birth of an affected child. Pregnancy rate increases are consistently being reported more often when PGS is used however we still do not know if or when it is safe to transfer embryos with some level of postzygotic aneuploidy. Aneuploidy screening is of course only one of 
several selection strategies for assessing and determining embryos for transfer. All will require further review, ensuring the highest possible chances of IVF. It is undeniable that further research in the future is required to optimize PGS for clinical use. For instance, we need to understand mosaicism better; where a nonmosaic euploid embryo is available this will always be the first choice for transfer, but where mosaic embryos are all that is available we need to better understand under what circumstances these are safe to transfer. We need to understand the origin of trisomies; embryos with trisomies that are meiotic in origin should not be transferred, however we need to be better informed when detecting if a trisomy is postzygotic and the clinical outcome this will lead to, if transferred.

A final question therefore is: if PGS can be demonstrated to increase IVF significantly, should it be offered to all IVF patients? While aneuploidy is more prevalent among patients of AMA, there are still many embryos that are aneuploid in younger patients. Moreover, as previously mentioned, CNVs can affect any age group of patients, PGS optimized for CNV screening can be used to benefit all patients of any age. Such a suggestion is likely to be contentious, particularly among the opponents of PGS. In any event, the debate for and against PGS is likely to rage for some time yet. A consideration rarely aired however is the issue of whether it is unethical not to offer PGS, given its potential benefits.

Open Access This article is distributed under the terms of the Creative Commons Attribution 4.0 International License (http://crea tivecommons.org/licenses/by/4.0/), which permits unrestricted use, distribution, and reproduction in any medium, provided you give appropriate credit to the original author(s) and the source, provide a link to the Creative Commons license, and indicate if changes were made.

\section{References}

1. HFEA. PGD conditions licensed by the HFEA. 2017. http:// guide.hfea.gov.uk/pgd/. Accessed 31 Jan 2017.

2. Gardner RL, Edwards RG. Control of the sex ratio at full term in the rabbit by transferring sexed blastocysts. Nature. 1968;218:346-8.

3. Steptoe PC, Edwards RG. Birth after the reimplantation of a human embryo. Lancet. 1978;2(8085):366.

4. Handyside AH, Kontogianni EH, Hardy K, Winston RML. Pregnancies from biopsied human preimplantation embryos sexed by Y-specific DNA amplification. Nature. 1990;344:768-70.

5. Handyside AH, Lesko JG, Tarín JJ, Winston RM, Hughes MR. Birth of a normal girl after in vitro fertilization and preimplantation diagnostic testing for cystic fibrosis. N Engl J Med. 1992;327:905-9.

6. Griffin DK, Wilton LJ, Handyside AH, Atkinson GHG, Winston RML, Delhanty JDA. Diagnosis of sex in preimplantation embryos by fluorescent in situ hybridisation. BMJ. 1993;306(6889):1382.

7. Delhanty JDA, Griffin DK, Handyside AH, Harper J, Atkinson GHG, Pieters MHEC, et al. Detection of aneuploidy and chromosomal mosaicism in human embryos during preimplantation sex determination by fluorescent in situ hybridisation, (FISH). Hum Mol Genet. 1993;2(8):1183-5.

8. Schrurs BM, Winston RML, Handyside AH. Preimplantantion diagnosis of aneuploidy using fluorescent in situ hydridization: evaluation using a chromosome 18-specific probe. Hum Reprod. 1993;8(2):296-301.

9. Munne S, Lee A, Rosenwaks Z, Grifo J, Cohen J. Diagnosis of major chromosome aneuploidies in human preimplantation embryos. Hum Reprod. 1993;8(12):2185-91.

10. Gianaroli L, Magli MC, Ferraretti AP, Tabanelli C, Trengia V, Farfalli V, et al. The beneficial effects of preimplantation genetic diagnosis for aneuploidy support extensive clinical application. Reprod BioMed Online. 2005;10(5):633-40.

11. Schoolcraft WB, Katz-Jaffe MG, Stevens J, Rawlins M, Munne S. Preimplantation aneuploidy testing for infertile patients of advanced maternal age: a randomized prospective trial. Fertil Steril. 2009;92:157-62.

12. Mastenbrook S, Twisk M, van Echten-Arends J, Sikkema-Raddatz B, Korevaar JC, Verhoeve HR, et al. In vitro fertilization with preimplantation genetic screening. $\mathrm{N}$ Engl $\mathrm{J}$ Med. 2007;357(1):9-17.

13. Kokkali G, Traeger-Synodinos J, Vrettou C, Stavrou D, Jones GM, Cram DS, et al. Blastocyst biopsy versus cleavage stage biopsy and blastocyst transfer for preimplantation genetic diagnosis of $\beta$-thalassaemia: a pilot study. Hum Reprod. 2007;22(5):1443-9.

14. Harton GL, Munne S, Surrey M, Grifo J, Kaplan B, McCulloh $\mathrm{DH}$, et al. Diminished effect of maternal age on implantation after preimplantation genetic diagnosis with array comparative genomic hybridization. Fertil Steril. 2013;100(6):1695-703.

15. Capalbo A, Ubaldi FM, Cimadomo D, Maggiulli R, Patassini C, Dusi L, et al. Consistent and reproducible outcomes of blastocyst biopsy and anueploidy screening across different biopsy practitioners: a multicentre study involving 2586 embryo biopsies. Hum Reprod. 2015;31(1):199-208.

16. Handyside AH, Robinson MD, Simpson RJ, Omar MB, Shaw MA, Grudzinskas JG, et al. Isothermal whole genome amplification from single and small numbers of cell: a new era for preimplantation genetic diagnosis of inherited disease. Mol Hum Reprod. 2004;10(10):767-72.

17. Wells D, Sherlock JK, Handyside AH, Delhanty JDA. Detailed chromosomal and molecular genetic analysis of single cells by whole genome amplification and comparative genomic hybridisation. Nucleic Acids Res. 1999;27(4):1214-8.

18. Rubio C, Rodrigo L, Mir P, Mateu E, Peinaddo V, Milan M, AlAsmar N, Campos-Galindo I, Garcia S, Simon C. Use of array comparative genomic hybridization (array-CGH) for embryo assessment: clinical results. Fertil Steril. 2013;99:1044-8.

19. Verlinsky Y, Rechitsky S, Schoolcraft W, Strom C, Kuliev A. Preimplantation diagnosis for fanconi anemia combined with HLA matching. JAMA. 2001;285:3130-3.

20. Rechitsky S, Kuliev A, Sharapova T, Laziuk K, Ozen S, Barsky I, et al. Preimplantation HLA typing with aneuploidy testing. Reprod BioMed Online. 2006;12(1):89-100.

21. Lee E, Illingworth P, Wilton L, Chambers GM. The clinical effectiveness of preimplantation genetic diagnosis for aneuploidy in all 24 chromosomes (PGD-A): systematic review. Hum Reprod. 2015;30:473-83.

22. Handyside AH, Harton GL, Mariani B, Thornhill AR, Affara N, Shaw M-A, et al. Karyomapping: a universal method for genome wide analysis of genetic disease based on mapping crossovers between parental haplotypes. J Med Genet. 2010;47:651-8.

23. Natesan SA, Handyside AH, Thornhill AR, Ottolini CS, Sage K, Summers MC, et al. Live birth after PGD with confirmation by a comprehensive approach (karyomapping) for simultaneous 
detection of monogenic and chromosomal disorders. Reprod BioMed Online. 2014;29:600-5.

24. Kung A, Munné S, Bankowski B, Coates A, Wells D. Validation of next-generation sequencing for comprehensive chromosome screening of embryos. Reprod BioMed Online. 2015;31:760-9.

25. Fan J, Wang L, Wang H, Ma M, Wang S, Liu Z, et al. The clinical utility of next generation sequencing for identifying chromosome disease syndromes in human embryos. Reprod BioMed Online. 2015;31:62-70.

26. Roque M, Lattes K, Serra S, Psych ISB, Geber S, Carreras R, et al. Fresh embryo transfer versus frozen embryo transfer in in vitro fertilization cycles: a systematic review and meta-analysis. Fertil Steril. 2013;99:156-62.

27. Mawell SM, Colls P, Hodes-Wertz B, McCulloh DH, McCaffrey C, Wells D, et al. Why do euploid embryos miscarry? A casecontrol study comparing the rate of aneuploidy within presumed euploid embryos that resulted in miscarriage or live birth using next-generation sequencing. Fertil Steril. 2016;106(6):1414-9.

28. Alfarawati S, Fragouli E, Colls P, Wells D. Embryos of robertsonian translocation carriers exhibit a mitotic interchromosomal effect that enhances genetic instability during early development. PLoS Genet. 2012;8(10):e1003025. 